\title{
Erratum to: A Highly Stable Source of Spectral Lines
}

\author{
V. K. Bityukov ${ }^{a}$, R. A. Gorbunov ${ }^{b}$, D. S. Simachkov ${ }^{a, *}$, and A. V. Frunze ${ }^{a, c}$ \\ ${ }^{a}$ Federal State Budget Educational Institution of Higher Education MIREA-Russian Technological University, \\ Moscow, 119454 Russia \\ ${ }^{b}$ Rostest-Moscow, Moscow, 117418 Russia \\ ${ }^{c}$ Nonprofit Scientific and Technical Partnership Termokont, Moscow, 119361 Russia \\ *e-mail: simachkov@mirea.ru \\ Received May 29, 2019
}

DOI: $10.1134 / \mathrm{S} 0030400 X 19050266$ lows:

The ACKNOWLEDGMENTS should read as fol-

The results, presented in this article, have been obtained within the framework of the State Assignment of the Ministry of Education and Science of Russia (Assignment No. 8.5577.2017/8.9) for imple- mentation of the project on the subject "Study of noise characteristics and pulsations of microcircuits of mobile sources of secondary power supply".

The original article can be found online at https://doi.org/10.1134/S0030400X19040040. 